\title{
Evaluation of Bed Cover Properties Produced from Double Fabric Based on Honeycomb
}

\author{
A. A. Salama, A. S. El-Deeb, and I. M. El-shahat \\ Textile Engineering Department, Mansoura University, Mansoura 35516, Egypt \\ Correspondence should be addressed to A. A. Salama; eng_amanysalama@yahoo.com
}

Received 20 September 2014; Accepted 4 March 2015

Academic Editor: Sambandam Anandan

Copyright (C) 2015 A. A. Salama et al. This is an open access article distributed under the Creative Commons Attribution License, which permits unrestricted use, distribution, and reproduction in any medium, provided the original work is properly cited.

\begin{abstract}
This research aims to innovate a new fabric structure, which could be used as a bed cover based on double honeycomb fabric with self-stitching. The honeycomb air pockets were aimed at facing each other to form closed small air chambers which work to sequester the air. The double fabric increases fabric thickness. Thus, the opportunity to improve thermal comfort could be achieved. A number of samples were produced with different densities and counts of weft yarn. Thermal insulation and water vapour permeability were measured and compared with bed covers produced from reversible weft backed structure. Geometrical properties, abrasion resistance, and air permeability were also measured. The results showed that the innovated structure had higher values of thermal insulation than reversible weft backed structure at certain weft counts and densities.
\end{abstract}

\section{Introduction}

Clothing is considered as a second skin of the human body as it works to protect the human body from hazardous substances [1]. It also controls the thermal equilibrium of man in his environment and preserves the human body from losing its temperature, since fabric organizes the heat transfer and gas exchange between the human body and the ambient atmosphere in different environmental conditions.

Comparing awaking state to sleep state, we can find that the energy emitted by the human body drastically reduces from 1500 watts as a maximum limit to 100 watts as a minimum limit during sleep [2]. Therefore, extra textiles are needed to achieve thermal equilibrium. These textiles are called bed covers, which support clothing in organizing heat and gas exchange. The main required properties of bedcovers are thermal insulation and water vapour permeability in addition to a reasonable weight.

These types of textiles vary according to the abovementioned properties from light bed sheets to heavy blankets and medium weight coverlet which is considered to be the most suitable bed covers in mid seasons.

The main traditional fabric structures of Egyptian coverlets are reversible weft backed, single honeycomb, and wadded double plain weave with interchangeable layers.
This research is an attempt to innovate and evaluate a fabric structure that could be used to produce a coverlet and compare its thermal properties to reversible weft backed coverlet made from the same warp and weft yarns.

\section{Review of Literature}

Wilson et al. described variables affecting thermal resistance of infant bedding materials such as different types of bedding, different sleep positions, methods of tucking, and two types of mattress. It was found that thermal resistance of bedding increased as thickness of bedding increased [3]. Fukazawa et al. measured thermal and water-vapour resistances of three kinds of cold-protective clothing ensembles in a cold environment. Sweating rate affected skin wittedness [4]. Qian and Fan showed that the surface thermal insulation is little affected by moisture transfer by using the sweating fabric manikin-Walter under varying climatic conditions and walking speeds [5]. Lin and Deng measured the total insulation values for a wide range of bedding systems. The insulation values were influenced by bedding and its percentage coverage over a human body and sleepwear [6]. Matusiak and Sikorski produced 19 variants of cotton woven fabrics of different weaves, linear densities of the weft, and different 


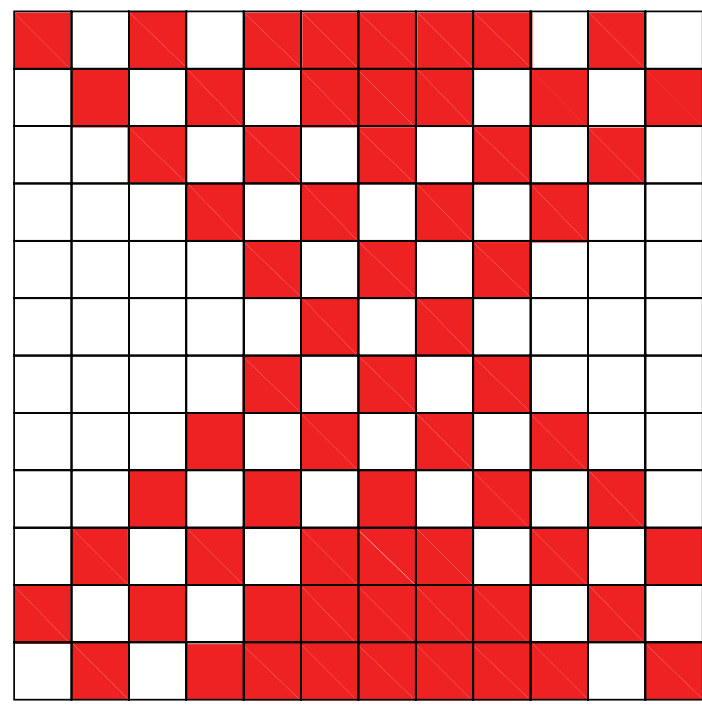

(a) Face structure

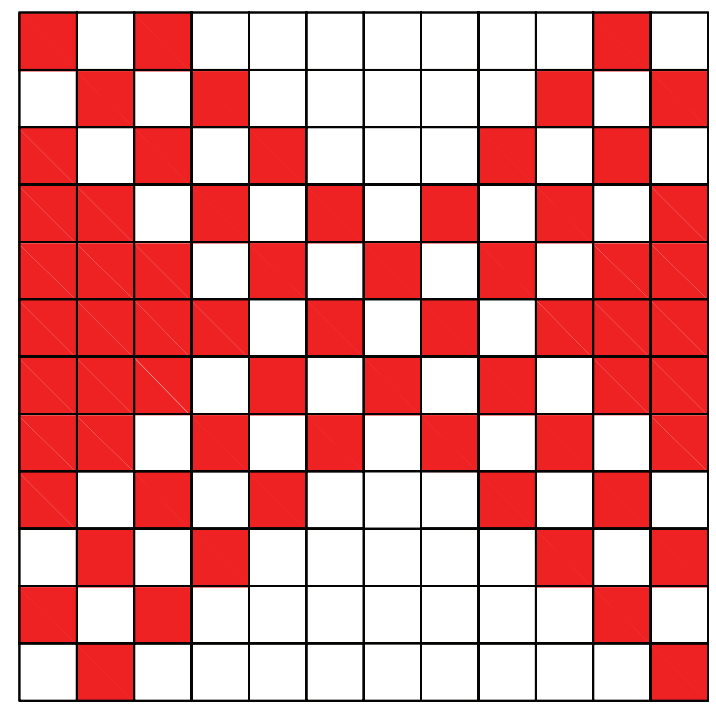

(b) Back structure

FIGURE 1: Face and back structure.

weft densities. On the basis of the results, the weave of woven fabrics, the linear density of weft yarn, and fabric thickness influence their thermal insulation properties significantly. The mass per square meter of fabrics affected their thermal conductivity. Closed air inside the fabric structure plays the role in thermal insulation. A strong and statistically significant correlation exists between the thermal insulation properties of woven fabrics and their structural parameters [7].

The previous researches showed that each of the fiber type, yarn structure, yarn count, yarn density, fabric structure, and type of finishing affects fabric thermal properties. Also the increase of fabric thickness which works to lock up the air inside the weave plays an important role in fabric thermal insulation especially in bed covers [3].

The innovated structure which this research introduces is based on honeycomb to benefit from the fabric thickness and air pockets and producing double honeycomb so that air pockets can face each other to form air chambers. The effect of weave structure at different densities and counts of weft yarns on the thermal insulation of the double honeycomb was studied and compared to reversible weft backed weave based on twill 3/1 which is commonly used in the Egyptian environment.

\section{Design of the Innovated Structure}

Double honeycomb structure was designed with self-stitching where the face weave was honeycomb repeated on $(12 \times$ 12) as shown in Figure 1(a). The back weave was the same structure but it was shifted half repeat warp direction and half repeat weft direction as shown in Figure 1(b).

The arrangement of warp and weft threads for face and back was $(1: 1)$. Self-stitching was carried out diagonally on both sides of fabric by dropping a face end under a back pick and lifting a back end over a face pick. By this arrangement

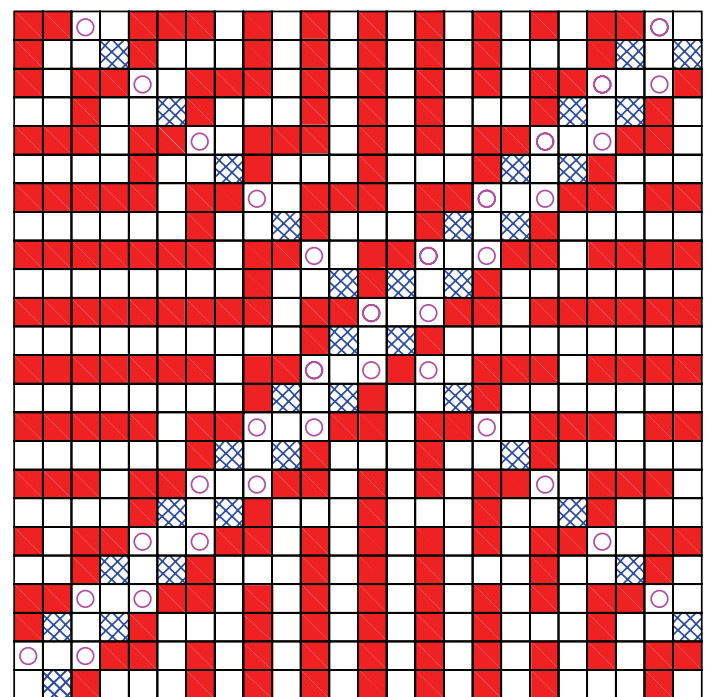

Lifting a back end over a face pick

0 Dropping a face end under a back pick

FIgURE 2: Self-stitched double honeycomb structure.

the air pockets in the face fabric will face the air pockets in the back fabric hopefully to form closed air chambers. Figure 2 shows one complete repeat $(24 \times 24)$ based on face and back weaves of Figure 1.

\section{Experimental Work}

Ten samples of the innovated double honeycomb weave were produced with two levels of weft density (16 and 22 p.p.cm) and each level of weft density was produced with five weft yarn counts $(14,12,10,8$, and $6 \mathrm{Ne})$. For the sake 


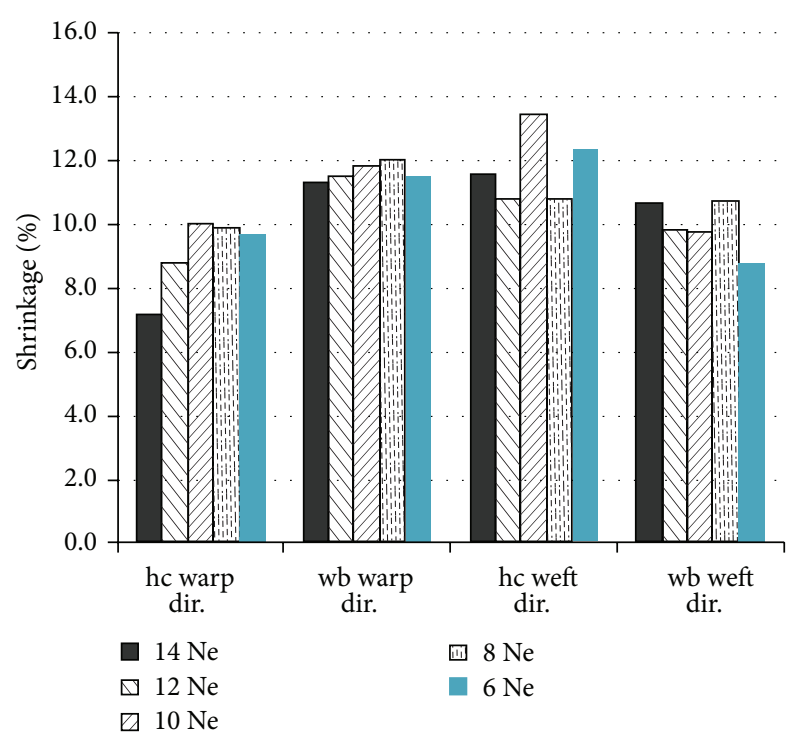

(a) For weft density 16 p.p.cm

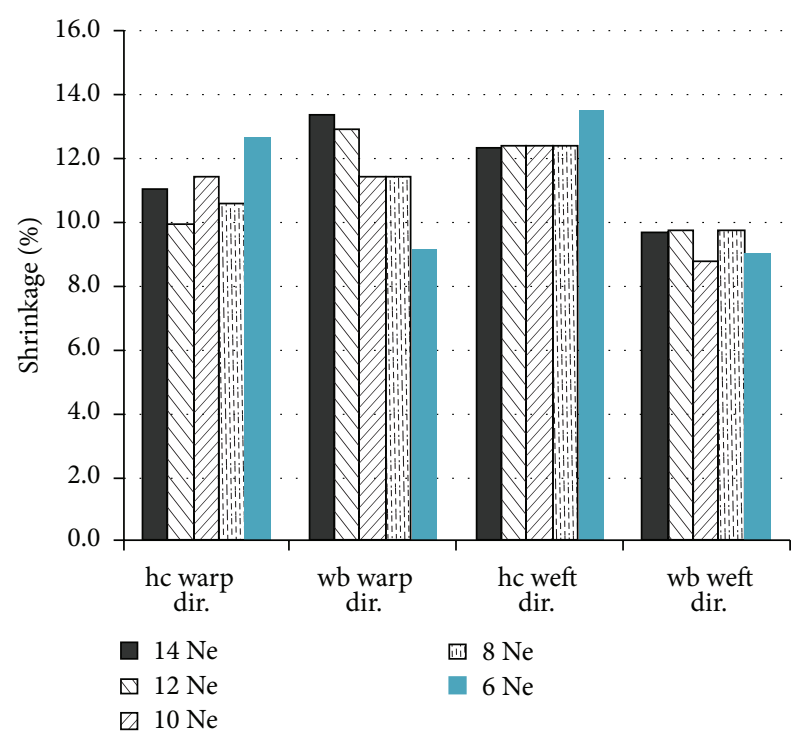

(b) For weft density 22 p.p.cm

FIGURE 3: Shrinkage percent for double honeycomb and reversible weft backed weave in warp and weft direction at two levels of pick density.

of comparison, other ten samples of reversible weft backed fabric were produced with the same previous densities and counts of weft yarn. All samples were produced from the same warp $(24 / 2 \mathrm{Ne})$ and $(24$ ends per $\mathrm{cm})$ on sulzertextil TPS 600 weaving machine. The two structures were designed with almost equal average float.

All samples were washed three cycles by using normal cotton program at a temperature of $90^{\circ} \mathrm{C}$. Geometrical and physical properties were measured after complete fabric relaxation. Thickness was measured according to ASTM D1777. Heat insulation was measured according to ASTM D 1518. Air permeability was measured according to ASTM D 737. Fabric stiffness was measured according to ASTM D4032. Abrasion resistance was measured according to ASTM D 3885. Water vapor permeability was measured according to ASTM E 96. Then, relative water vapor permeability (RWVP) was determined from the following equation:

$$
\operatorname{RWVP}(\%)=\frac{G_{S} * 100}{G_{o}},
$$

where $G_{S}=$ weight change with sample; $G_{o}=$ weight change without sample.

The crimp percent, shrinkage percent, and the fabric weight per unit area were measured, and bulk density was calculated from the following formula:

$$
\text { Bulk density }\left(\mathrm{kg} / \mathrm{m}^{3}\right)=\frac{W\left(\mathrm{~g} / \mathrm{m}^{2}\right)}{t(\mathrm{~mm})},
$$

where $W=$ fabric weight per unit area; $t=$ fabric weight.

\section{Experimental Results and Discussions}

Statistical analysis of all test results of physical and geometrical properties mentioned above was carried out by using
Multiway ANOVA by using SPSS program to examine the significance of the structure, density, and count of weft yarns on these properties.

5.1. Shrinkage. Figures 3(a) and 3(b) show the shrinkage percent of double honeycomb and reversible weft backed weave for 16 and 22 p.p.cm, respectively, for both warp and weft direction.

At the same count and density, shrinkage percent of double honeycomb in warp direction (hc warp dir.) is less than reversible weft backed weave (wb warp dir.) and vice versa concerning the weft direction since the shrinkage percent of double honeycomb (hc weft dir.) is higher than the reversible weft backed weave (wb weft dir.). Statistical analysis showed a significant effect of weave structure on shrinkage percent in each of the warp and weft directions, but there is no significant influence of each of the count and density of the weft thread.

5.2. Fabric Thickness. Figure 4 presents the effect of weft count on the thickness of both double honeycomb (hc) and reversible weft backed (wb) samples at weft density of 16 and 22 p.p.cm. Generally, the thickness of double honeycomb fabric is higher than the reversible weft backed with a maximum of $77 \%$ at 22 p.p.cm and weft count of $6 \mathrm{Ne}$ because of the surface nature of each layer of stitched double honeycomb fabric. The thickness also decreases when weft count increases for both structures because of the inversed relation between yarn count $(\mathrm{Ne})$ and its diameter. Statistical analysis showed a significant effect of fabric structure and weft count and insignificant effect of weft density on fabric thickness.

5.3. Crimp Percent. Figure 5 shows the effect of weft count at the two levels of weft densities on warp crimp percent for 


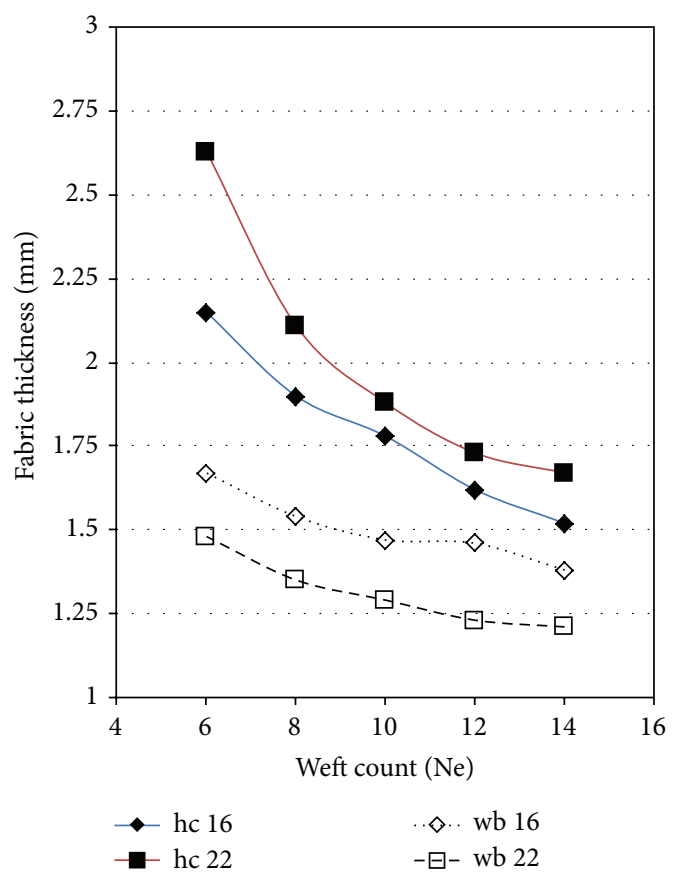

FIgURE 4: The effect of weft count on the thickness of both double honeycomb and reversible weft backed fabric at two weft densities.

both double honeycomb (hc) and reversible weft backed (wb) samples. The warp crimp percent decreased by weft count $(\mathrm{Ne})$ increasing for both structures by different rates as crimp percent is greatly affected by densities and counts of crosswise threads. The crimp percent of wb structure is higher than the crimp percent of hc structure probably because warp threads in case of wb structure interlaced between two levels of weft while warp threads of each layer in hc structure interlaced within the same layer except at stitching point. Statistical analysis showed that the effect of weft count and structure on warp crimp is significant.

Figure 6 shows a slight effect of weft count on weft crimp percent for both double honeycomb (hc) and reversible weft backed (wb) samples at the two levels of weft densities as the warp count and density are the same in all samples. The crimp percent of hc sample is higher than the wb sample crimp percent. Statistical analysis showed a significant effect of fabric structure and insignificant effect of weft count on the weft crimp.

The warp crimp increases at the expense of weft crimp in the case of the reversible weft backed sample, while the weft crimp increases at the expense of warp crimp in the case of the double honeycomb sample. This is due to the crimp interchange; that is, the crimp of one direction increases at the expense of the other direction.

5.4. Fabric Density. Figure 7 presents the effect of weft count on fabric weight on both double honeycomb (hc) and reversible weft backed (wb) samples at two weft densities.

At the same weft count and density, the weight per unit area in both structures is almost the same which means that the production cost of each structure is the same. Statistical

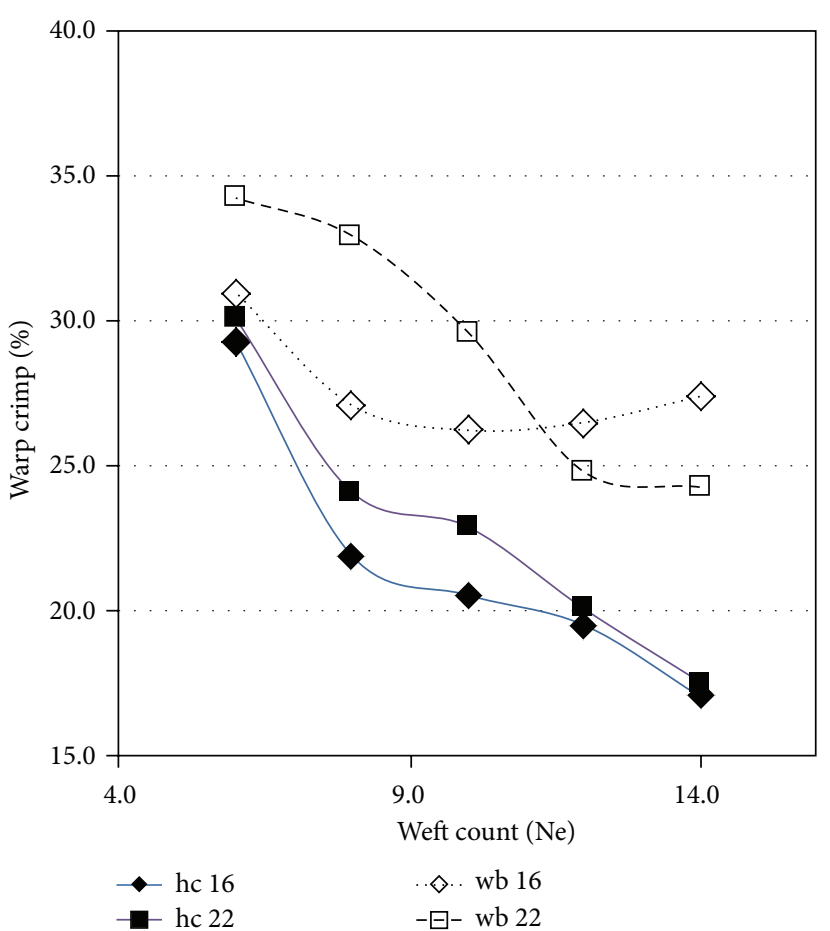

FIGURE 5: Warp crimp percent for double honeycomb and reversible weft backed samples.

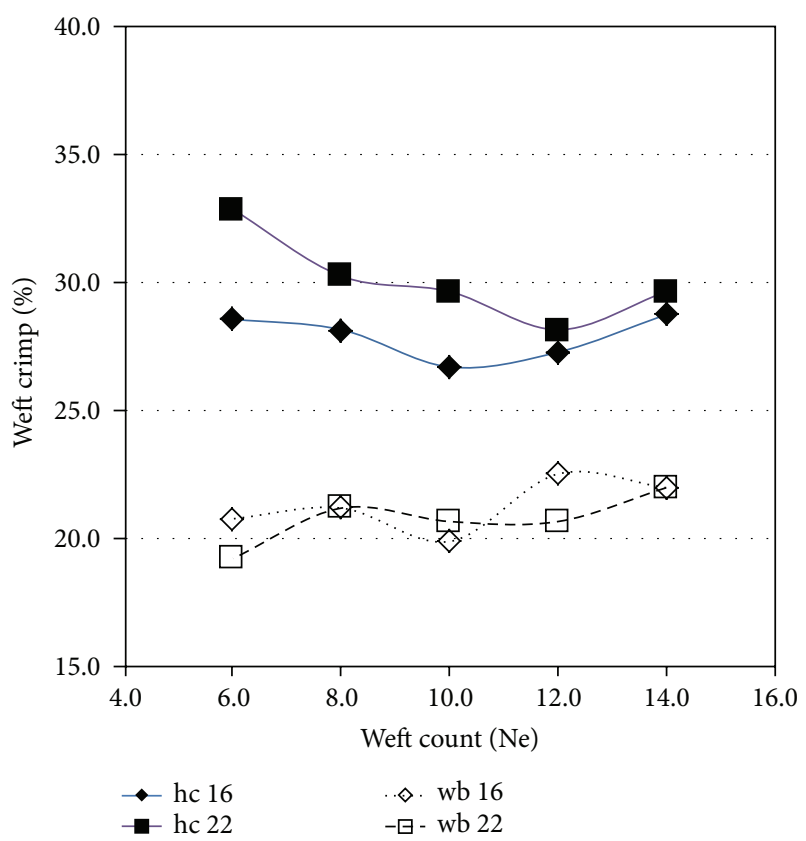

FIgURE 6: Weft crimp percent for double honeycomb and reversible weft backed samples.

analysis showed that the effect of structure on fabric weight is insignificant.

5.5. Fabric Bulk Density. Figure 8 shows the effect of weft count on the bulk density for double honeycomb (hc) and reversible weft backed $(\mathrm{wb})$ samples at two weft densities. The 


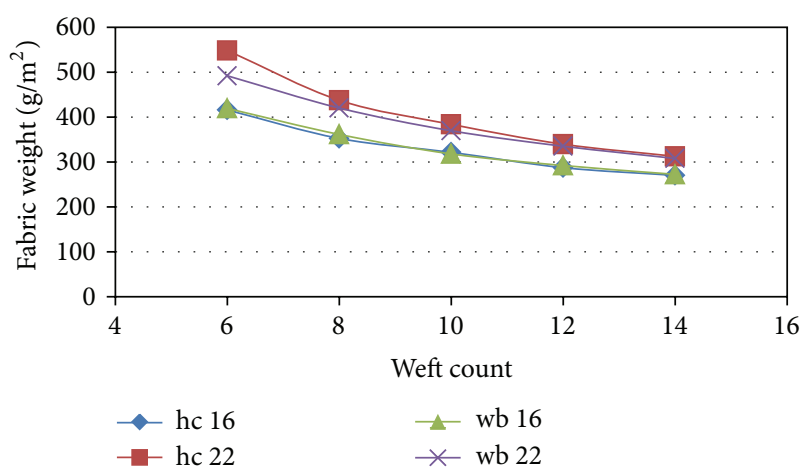

Figure 7: The effect of weft count on fabric weight of double honeycomb and reversible weft backed samples at two weft densities.

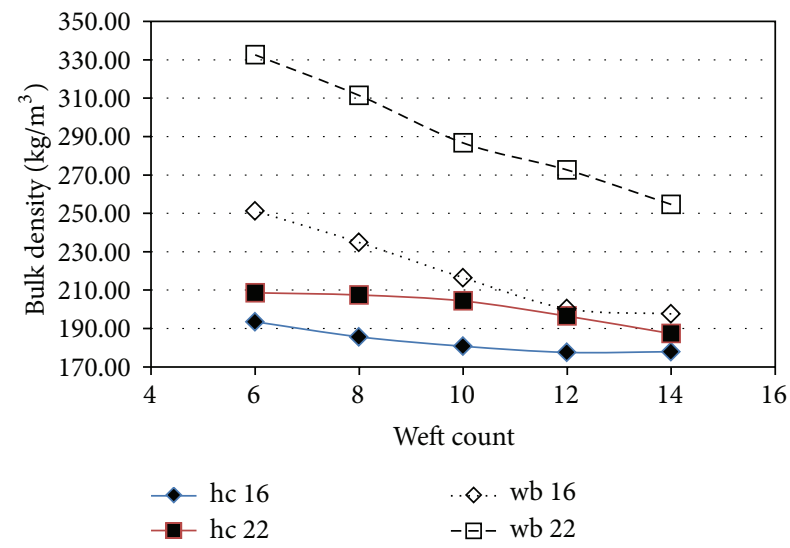

FIGURE 8: The effect of weft count on the bulk density of double honeycomb and reversible weft backed samples at two weft densities.

bulk density of the weft backed cloth is higher than the double honeycomb at the same weft count and density. Therefore, the amount of air trapped inside the double honeycomb fabric is higher than weft backed cloth and this may lead to an expected increase of thermal insulation.

5.6. Heat Insulation. Figure 9 shows a fluctuation effect of weft count on thermal insulation for both double honeycomb (hc) and reversible weft backed (wb) samples at 16 p.p.cm. Thermal insulation of hc fabric increases by $59 \%$ compared to weft backed fabric at count $10 \mathrm{Ne}$.

Figure 10 shows also a fluctuation effect of weft count on thermal insulation for both double honeycomb (hc) and reversible weft backed (wb) samples at 22 p.p.cm. Thermal insulation of hc fabric is higher than wb fabric at count $12 \mathrm{Ne}$ by $49 \%$ and at count $8 \mathrm{Ne}$ by up to $43 \%$.

The thermal insulation decreases by an average of $19 \%$ in both structures with increasing of weft density, as the bulk density $\left(\mathrm{kg} / \mathrm{m}^{3}\right)$ for the produced samples from density 16 p.p.cm is less than the produced samples from density 22 p.p.cm by an average of $9 \%$ in honeycomb and by an average of $24 \%$ in weft backed sample. Therefore the produced samples with lower density (16 p.p.cm) contain more air than the produced samples with the highest density (22 p.p.cm);

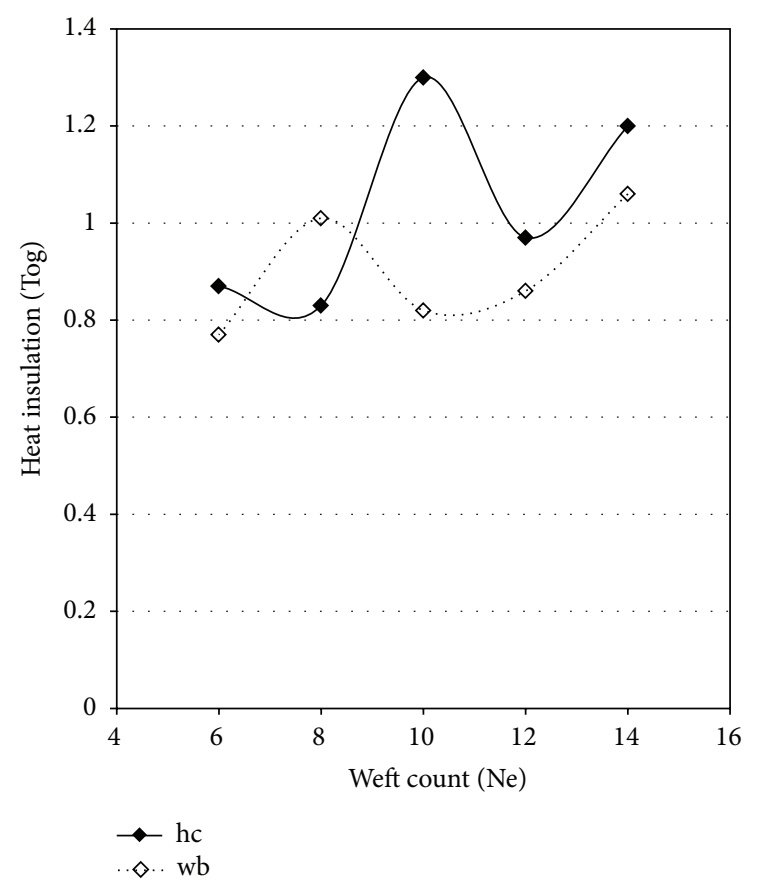

FIGURE 9: Effect of weft count on thermal insulation of double honeycomb and reversible weft backed samples at 16 p.p.cm.

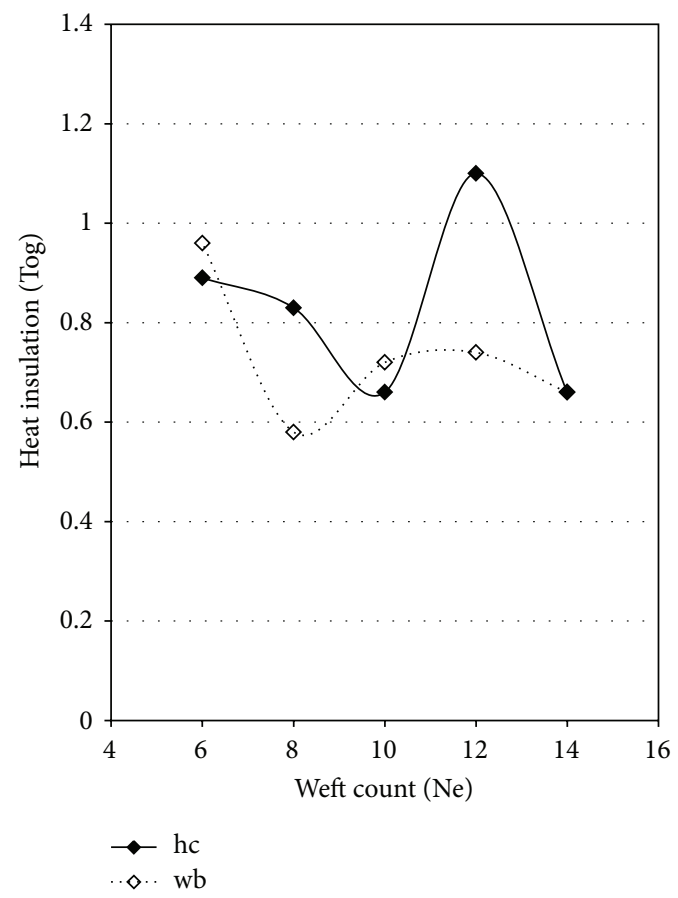

FIGURE 10: Effect of weft count on thermal insulation of double honeycomb and reversible weft backed samples at 22 p.p.cm.

therefore the opportunity to produce higher insulation at lower cost could be achieved. Statistical analysis shows a significant effect of structure, weft count, and weft density on the thermal insulation. So, there are certain values of weft density and count of double honeycomb fabric to obtain 


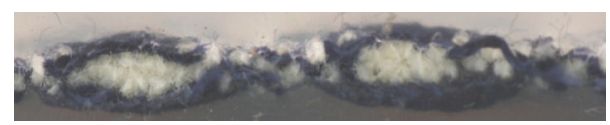

(a) Warp cross section

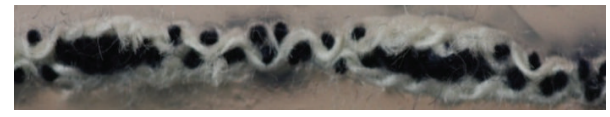

(b) Weft cross section

FIGURE 11: Cross section in double honeycomb fabric.

higher thermal insulation values than reversible weft backed fabric.

Figures 11(a) and 11(b) show warp and weft cross sections for two successive repeats of self-stitched double honeycomb at weft density 16 p.p.cm and weft count $10 \mathrm{Ne}$, respectively, since maximum heat insulation was achieved. From both figures it is clear that the fabric is not of uniform thickness, that is, fabric of less thickness at stitching point and higher thickness at maximum float. It is also clear that both warp and weft spacing are not uniform, that is, widely spacing at stitching point and closer spacing at max float. Therefore, air pockets did not appear as expected at fabric zones of higher thickness and closer spacing which might reflect on thermal properties.

5.7. Relative Water Vapour Permeability. Figures 12(a) and 12(b) present the effect of fabric structure on the relative water vapor permeability at 16 and 22 p.p.cm, respectively. In spite of the presence of two layers of the double honeycomb fabric, the water vapor permeability was almost equal to the backed cloth. Statistical analysis showed that the effect of structure, count, and density is insignificant on the relative water vapor permeability. The increase of thermal insulation by $59 \%$ with maintaining water vapour permeability means better thermal comfort.

The most insulated double honeycomb sample (at 16 p.p.cm and weft yarn count of $10 \mathrm{Ne}$ ) was tested for abrasion resistance, fabric stiffness, crease resistance, and air permeability. The test results were compared with reversible weft backed sample at the same weft yarn count and density. The abrasion resistance of double honeycomb fabric was higher than reversible weft backed fabric by $48.6 \%$ which could be interpreted by the honeycomb (hc) fabric that is not a uniform thickness (two levels at stitching points and at maximum float) while the thickness of weft backed (wb) fabric is a uniform thickness. Therefore, the area exposed to the abrasion in hc fabric is less than the area in wb fabric; so the weight loss due to abrasion for double fabric is less than the weight loss for backed fabric. Flexural rigidity of hc fabric was less than wb fabric by $52 \%$ in the weft direction while it increased by $40.5 \%$ in the warp direction. The crease resistance of hc fabric was less than wb fabric by $9.6 \%$ in the weft direction, while in the warp direction it decreased by $3.4 \%$. Air permeability for both structures was almost the same.

Therefore, from all samples and experimental tests, weft count of $10 \mathrm{Ne}$ and weft density of 16 p.p.cm were

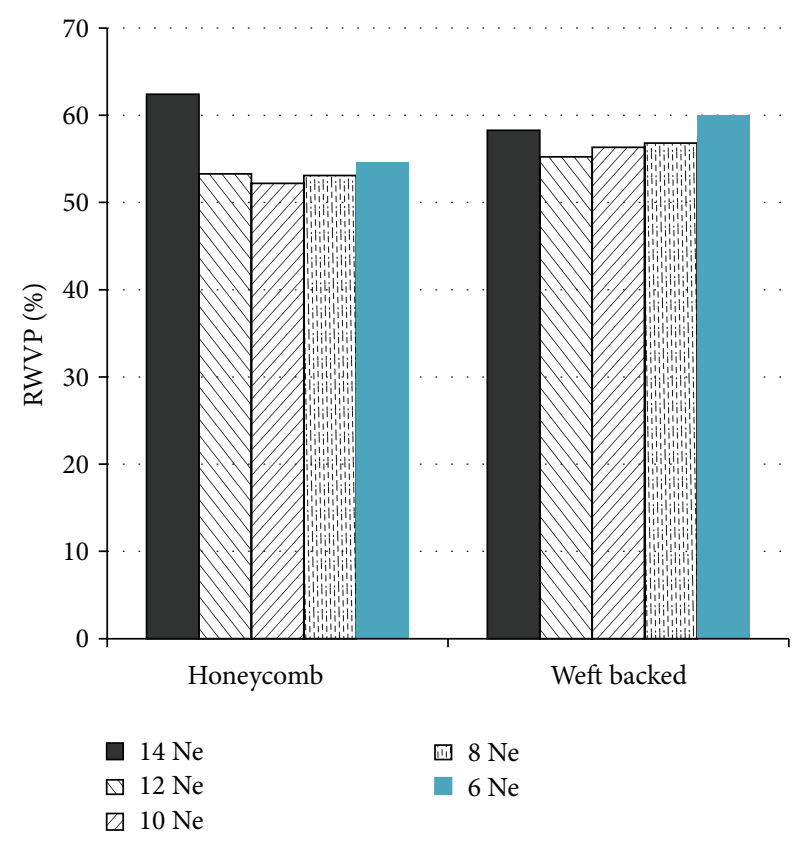

(a) For weft density 16 p.p.cm

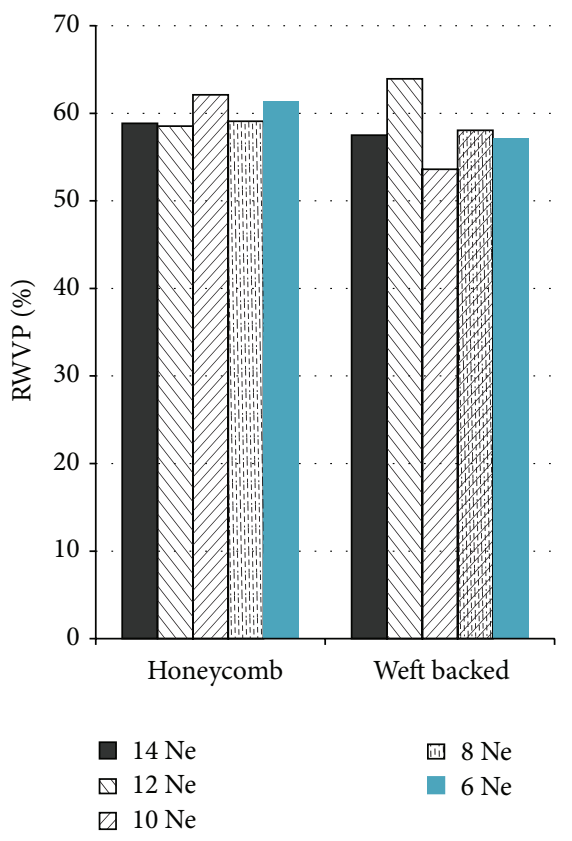

(b) For weft density 22 p.p.cm

FIGURE 12: Influence of double honeycomb and reversible weft backed weave on relative water vapor permeability at two levels of pick density.

recommended to produce a good bed cover from double honeycomb compared with reversible weft backed fabric made from the same weft yarn.

\section{Conclusion}

(i) An attempt to innovate a fabric structure that could be used to produce a coverlet based on self-stitched double honeycomb was made. 
(ii) The innovated structure was compared to reversible weft backed structure made from the same weft counts and densities.

(iii) Double honeycomb fabric has higher values of thermal insulation than reversible weft backed fabric at certain weft counts and densities.

(iv) In spite of the presence of two layers of double honeycomb, its water vapor permeability is almost equal to the reversible weft backed fabric.

(v) The thermal comfort of the innovated structure could be improved by using bulky filament yarns as wadded weft threads to pry the fabric layers.

\section{Conflict of Interests}

The authors declare that there is no conflict of interests regarding the publication of this paper.

\section{References}

[1] A. K. Haghi, S. Thomas, and L. A. Pothan, Heat and Mass Transfer in Textiles: Theory and Applications, WSEAS Press, 2007.

[2] B. P. Saville, Physical Testing of Textiles, North America by CRC Press LLC 2000 Corporate Blvd, NW, Boca Raton, Fla, USA, 2000.

[3] C. A. Wilson, R. M. Laing, and B. E. Niven, "Estimating dry thermal resistance of multiple-layer bedding materials-reexamining the problem," Journal of the Human-Environment System, vol. 2, no. 1, pp. 69-85, 1999.

[4] T. Fukazawa, G. Lee, T. Matsuoka, K. Kano, and Y. Tochihara, "Heat and water vapour transfer of protective clothing systems in a cold environment, measured with a newly developed sweating thermal manikin," European Journal of Applied Physiology, vol. 92, no. 6, pp. 645-648, 2004.

[5] X. Qian and J. Fan, "Interactions of the surface heat and moisture transfer from the human body under varying climatic conditions and walking speeds," Applied Ergonomics, vol. 37, no. 6, pp. 685-693, 2006.

[6] Z. Lin and S. Deng, "A study on the thermal comfort in sleeping environments in the subtropics-measuring the total insulation values for the bedding systems commonly used in the subtropics," Building and Environment, vol. 43, no. 5, pp. 905916, 2008.

[7] M. Matusiak and K. Sikorski, "Influence of the structure of woven fabrics on their thermal insulation properties," Fibres \& Textiles in Eastern Europe, vol. 19, no. 5 (88), pp. 46-53, 2011. 

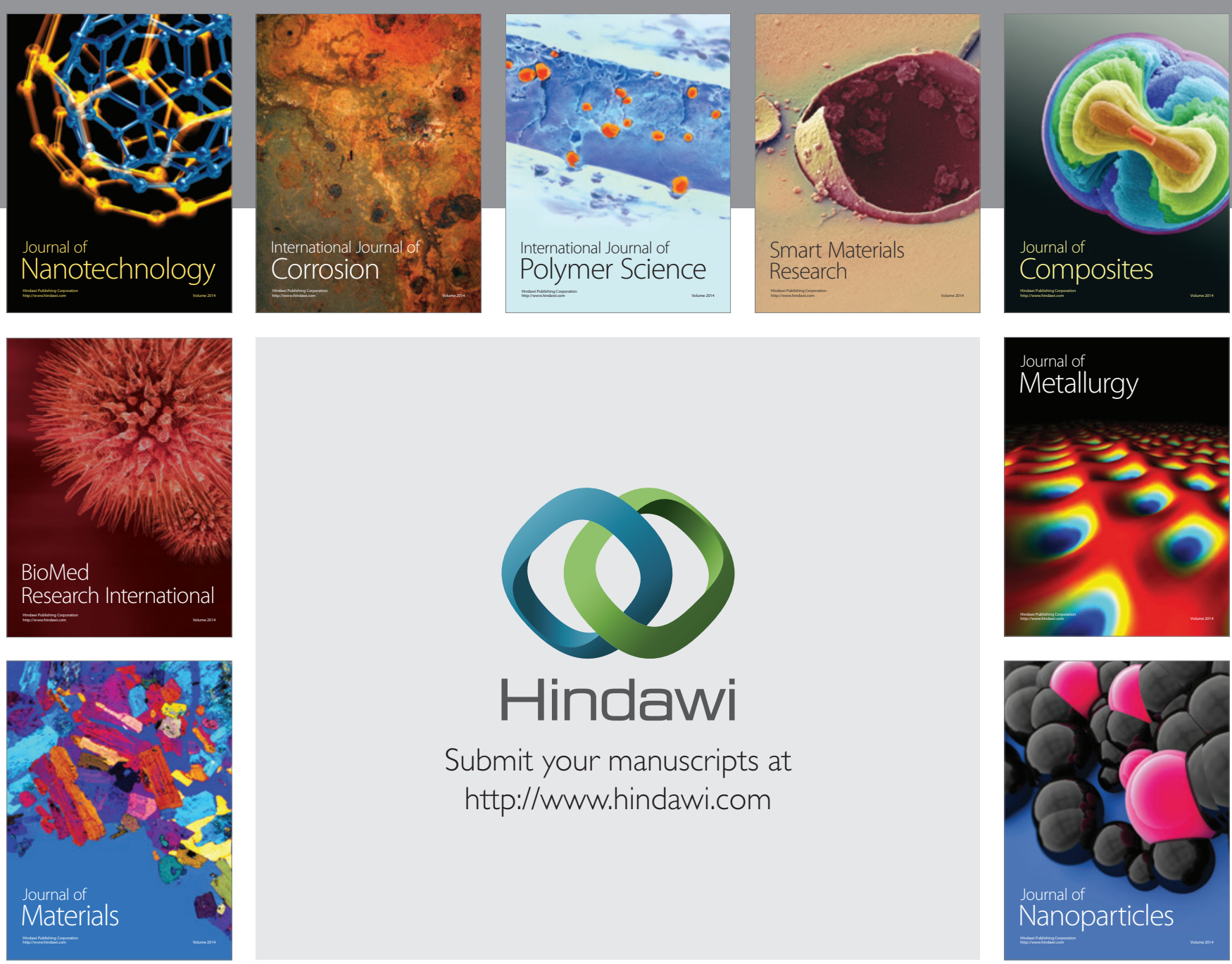

Submit your manuscripts at http://www.hindawi.com
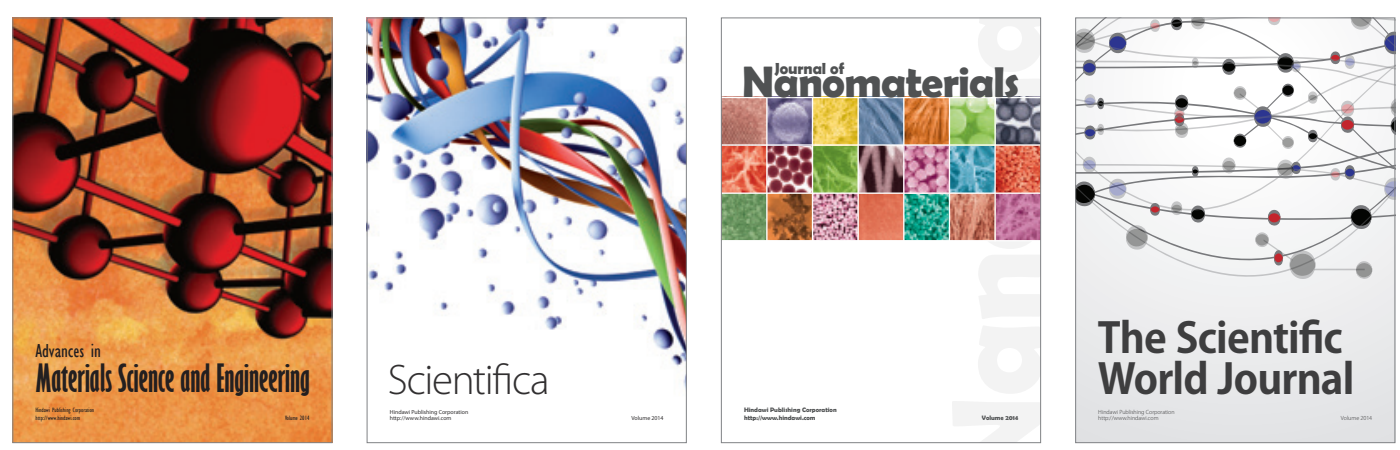

\section{The Scientific World Journal}
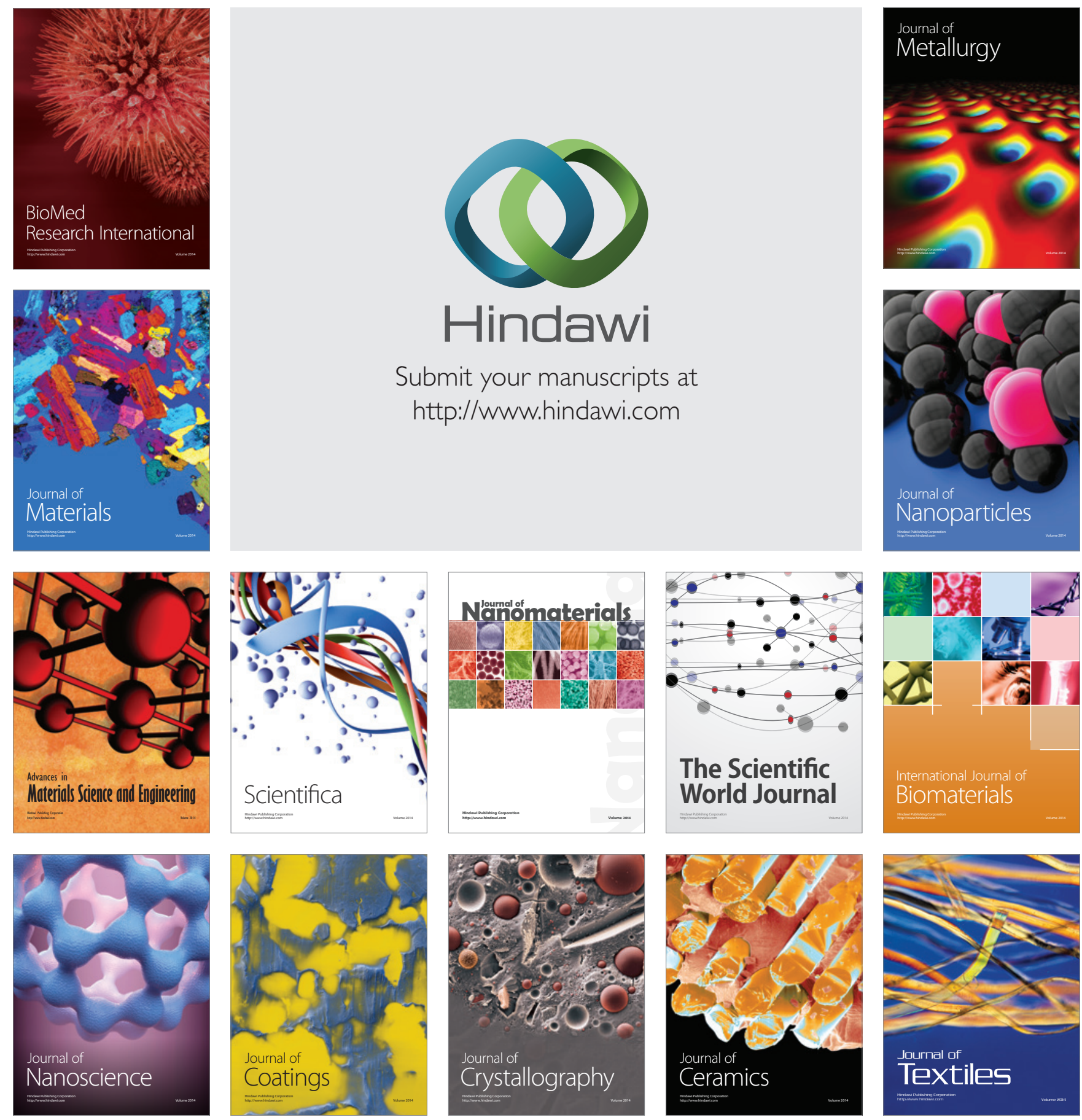